\title{
SAR-SIFT for Matching Multiple SAR Images and Radargrammetry
}

\author{
Clémence Dubois $^{1}$ - Andrea Nascetti ${ }^{2}$ - Antje Thiele ${ }^{3,4}$. \\ Mattia Crespi $^{2}$. Stefan Hinz ${ }^{3}$
}

Received: 1 April 2016 / Accepted: 1 March 2017 / Published online: 3 July 2017

(C) Deutsche Gesellschaft für Photogrammetrie, Fernerkundung und Geoinformation (DGPF) e.V. 2017

\begin{abstract}
This article presents a new application of the SAR-SIFT algorithm proposed by Dellinger et al. (IEEE Trans Geosci Remote Sens 53:453-466, 2015) for the automatic generation of tie points (TPs). In particular, SAR-SIFT is applied on stereo-SAR images to extract corresponding points and determine their 3D position. Furthermore, the potential of the combined use of the SAR-SIFT with the SAR-stereo model for extracting reliable TPs is analysed, by evaluating their absolute accuracy in $2 \mathrm{D}$ and $3 \mathrm{D}$. The tests are performed with TerraSAR-X HR SpotLight data acquired
\end{abstract}

Clémence Dubois

clemence.dubois@bgr.de

Andrea Nascetti

andrea.nascetti@uniroma1.it

Antje Thiele

antje.thiele@kit.edu; antje.thiele@iosb.fraunhofer.de

Mattia Crespi

mattia.crespi@uniroma1.it

Stefan Hinz

stefan.hinz@kit.edu

1 Department Geoscientific Information, International Cooperation, Sub-Department Geo-Hazard Assessment, Remote Sensing, Federal Institute for Geosciences and Natural Resources, 30655 Hannover, Germany

2 Department of Civil, Constructional and Environmental Engineeering, University of Rome 'La Sapienza', 00184 Rome, Italy

3 Institute of Photogrammetry and Remote Sensing, Karlsruhe Institute of Technology, 76131 Karlsruhe, Germany

4 Fraunhofer Institute of Optronics, System Technologies and Image Exploitation (IOSB), 76275 Ettlingen, Germany over Trento, Italy. Single and multiple image pairs have been considered. 3D accuracy assessment of the extracted points is performed relying on LiDAR data; the results show a planimetric accuracy up to $1 \mathrm{~m}$ and an elevation accuracy of about $2 \mathrm{~m}$.

Keywords SAR-SIFT applications - 2D/3D tie points . Accuracy assessment $\cdot$ Stereo $\cdot$ Radargrammetry

Zusammenfassung SAR-SIFT für das Matching von Multiplen SAR Bildern und Radargrammetry. In diesem Artikel wird eine neue Einsatzmöglichkeit mit dem Ziel der automatischen Extraktion von Verknüpfungspunkten (Tie Points: TPs) des SAR-SIFT-Algorithmus präsentiert, der von DELLINGER et al. (2015) vorgestellt wurde. Der SARSIFT-Algorithmus wird auf SAR-Stereobildpaare zur Extraktion homologer Punkte angewandt, deren 3D-Koordinaten mithilfe des SAR-Stereomodells ermittelt werden. Das Potential der kombinierten Anwendung des SAR-SIFT mit dem SAR-Stereomodell zur Extraktion zuverlässiger TPs wird anhand ihrer absoluten 2D/3D-Punktgenauigkeit untersucht. Die Untersuchungen werden auf TerraSAR-X HR-SpotLightDaten von Trento (Italien) durchgeführt. Sowohl einzelne als auch multiple radargrammetrische Bildpaare werden betrachtet. Die 3D-Genauigkeit der extrahierten Punkte wird anhand von LiDAR-Daten bewertet. Die Ergebnisse weisen eine Lagegenauigkeit von bis zu $1 \mathrm{~m}$ und eine Höhengenauigkeit von circa $2 \mathrm{~m}$ auf.

\section{Introduction}

In the last decade, Earth observation using high-resolution SAR missions underwent a high development. For example, TerraSAR-X, TanDEM-X, COSMO-SkyMed, RADARSAT, and so on, the TerraSAR-X-like new Spanish PAZ sensor, 
enable to acquire imagery with a resolution better than $1 \mathrm{~m}$. Using the new acquisition mode Staring SpotLight that has been available since 2013 on TerraSAR-X platforms, the resolution can even reach $0.25 \mathrm{~m}$ (Mittermayer et al. 2014). The resulting amplitude images feature much more details, which enables a multitude of new applications. Among these new applications, object detection, e.g., buildings, cars, ships, and airplanes, is a popular topic. Especially, structure analysis using the recognition of linear patterns and point features is of increasing interest. One of the major challenges is the recognition of such features in several images taken under different acquisition conditions, presenting different geometry and speckle noise. In particular, the extraction and matching of homologous point features in different SAR images are on focus. The SAR-SIFT algorithm, introduced by Dellinger et al. (2015), presents a major improvement towards this goal, permitting the highly accurate matching of homologous points and the subsequent coregistration of SAR images taken under different incidence angles. Until now, this new algorithm is used for $2 \mathrm{D}$ applications such as image coregistration or change detection.

However, from the possibility of matching keypoints between imagery taken with different incidence angles, a new 3D application arises, based on a radargrammetric approach. Indeed, using images taken from different incidence angles allows the determination of the absolute 3D point coordinates, which is otherwise not possible with only one SAR image, or with an interferometric pair that allows only the determination of a relative point height. The goal of this work is to analyse the capability of the SAR-SIFT algorithm for finding automatically corresponding tie points (TPs) and reconstructing their 3D position by radargrammetry. Such TPs are, for instance, useful for georeferencing SAR as well as optical images, for correcting locally and pointwise potential biases of extracted digital elevation models (DEMs), and for analysing terrain motion based on persistent scatterers (PS) extracted in the amplitude image (Nascetti et al. 2015a). Especially for PS interferometry, the 3D position of the TPs (in this context also often utilized as ground control points: GCPs) is often required to attach the linear motion model to known, stable points. Furthermore, as such points usually are strong scatterers, it is very likely that GCPs found using the SAR-SIFT algorithm correspond to some PS points. The automatic extraction of GCPs is of particular importance in areas, where no or only little ground truth, e.g., GPS measurement, is available.

In this paper, we evaluate the 2D and 3D accuracy of TPs estimated based on a combination of the stereo-SAR model and SAR-SIFT. For this purpose, we first present a basic approach for extracting the TPs. Then, we retain suitable points using some thresholds based on approximate height information and analyse their 3D accuracy, e.g., based on LiDAR data. This approach solely intends to give an indi- cation about the procedure and the accuracy potential of extracting 3D points based on matched 2D points. The steps that are conducted to eliminate obviously wrong TPs could be further improved to provide an even more reliable and robust TPs' extraction method. Furthermore, we assume in the following that the planimetric position of extracted 3D points situated in close vicinity of a distinct height discontinuity corresponds actually to the position of this height jump. Our goal here is rather to show the potential and the accuracy that can be achieved using a combination of the two methodsSAR-SIFT and Stereo-SAR based on the extracted TPs.

\section{State-of-the-Art}

Due to their very precise orbit information and slant-range measurement, SAR data are very valuable for the determination of absolute 3D information. Recently, different approaches have been developed for improving the georeferencing of optical imagery using SAR data.

The approach presented in Reinartz et al. (2011) supports orthorectification of optical imagery considering a set of TPs extracted from geocoded TerraSAR-X imagery. For this purpose, mutual information is used for the matching of corresponding optical and SAR patches. One drawback of this approach is the use of an external DEM for geocoding, which could induce a systematic bias in the absolute coordinates. Another approach (Koppe et al. 2012) performs TP/GCP extraction using the radargrammetric stereo model of TerraSAR-X image pairs, and assesses their accuracy using whether DGPS survey or LiDAR DEM as a reference. The GCPs are extracted manually, and the achieved 3D accuracy is up to $2 \mathrm{~m}$ for the same side, and up to $1 \mathrm{~m}$ for opposite-side stereo pairs. In Chureesampant and Susaki (2014), the authors extract TPs automatically from polarimetric SAR images using the standard SIFT algorithm (Lowe 1999) and evaluate the performance of the extraction using the signal-to-noise ratio (SNR). The TPs are extracted on L-band SAR data and only the accuracy of 2D matching between images has been estimated. However, the authors showed the capability of the SIFT algorithm to find similar features in images taken from different incidence angles.

To the best of our knowledge, at the time of writing this paper, there exists no approach presenting an evaluation of automatically extracted and (3D-) reconstructed TPs/GCPs from SAR images, while the matching of SAR images taken from different incidence angles has a long tradition. Several methods have been proposed, including pixel-based (e.g., sliding window) or feature-based approaches (e.g., SIFT). Pixel-based approaches produce satisfying results for dense matching estimates with small incidence angles. For larger differences of incidence angles between the SAR images, it is better to use feature-based approaches. Even if the SIFT 
algorithm can be used on SAR images, it suffers from the inherent speckle noise, and matching two SAR images relying on SIFT keypoints and descriptors provides much less matches than in optical images. For a few years, authors have tried to adapt the SIFT algorithm to SAR images, either using filtering methods (Liu and Yu 2008) or by modifying steps of the algorithm (Suri et al. 2010). The SAR-typical SIFT algorithm (SAR-SIFT) used in this work fits within the latter group. It was developed by Dellinger et al. (2015), who also give an exhaustive comparison with other SAR-adapted SIFT approaches. The main difference compared to the standard SIFT algorithm of Lowe (1999) consists of the definition of a new method for gradient computation, called gradient by ratio (GR), based on the logarithm of the ratio of exponentially weighted average (ROEWA), defined in Fjortoft et al. (1998). This new gradient operator considers ratio of intensities instead of intensity differences and, therefore, presents several advantages. First, it is more robust to speckle noise, and second, it allows a reliable estimation of the gradient in multiple directions and image scales. The standard SIFT algorithm is modified at several steps considering this new gradient operator, which allows to enhance its performance for SAR images. First, for the keypoint detection, new multiscale SAR-Harris matrix and function are defined, using the GR instead of the second-order derivatives of the Gaussian filtered intensity values. Instead of using the original Laplacian of Gaussian (LoG) image scale space for the keypoint detection, a new multiscale image representation is defined using the weighting parameter of the ROEWA as scale parameter. Detected keypoints are situated on corners, edges, and salient point features, proving the robustness of the method against false detections caused by speckle. Second, the GR is used for the computation of the orientation histograms at each keypoint. For this purpose, a scale-dependent circular neighbourhood is defined around each keypoint. Finally, keypoint descriptors are extracted by concatenating the gradient orientations defined by GR in polar sectors of a circularscale-dependent neighbourhood.

Considering an image pair, keypoints extracted in both images can be matched using their respective descriptors. Matches are found by computing nearest neighbour (NN) or nearest neighbour distance ratio (NNDR). For all criteria, the Euclidean distances between the descriptors of both images are computed. For the NN criterion, the descriptor of the second image showing the smallest distance to the one of the first image is retained. The NNDR criterion allows to filter wrong matches by calculating the ratio of distances of the closest and second closest descriptors of the second image to a specific descriptor of the first image. A threshold is defined on this ratio for deciding to keep the point as matched point or to reject it. In Dellinger et al. (2015), only $\mathrm{NN}$ is applied for images taken with different incidence angles.
The performance of SAR-SIFT has been evaluated in Dellinger et al. (2015) considering different image resolutions and different incidence angles. Several TerraSAR-X and COSMO-SkyMed data sets were used for assessing the performance of SAR-SIFT on different image resolutions. Furthermore, coregistration of images presenting different incidence angles has been performed with COSMO-SkyMed data, with subsequent quality evaluation. For determining the image transformation between the images, RANSAC was used in Dellinger et al. (2015) to eliminate wrong matches.

In our approach, we rely on a modification of the described SAR-SIFT algorithm for extracting relevant feature points. By matching homologous points from images acquired from different incidence angles, it is possible to extract their 3D coordinates using a radargrammetric approach. Of course, the 2D localisation accuracy of the feature points is an important aspect for matching, yet the final 3D position determined by radargrammetry is the ultimate goal in our evaluation .

Section 3 explains the methodology starting with an overview of the workflow (Sect. 3.1). In Sect. 3.2, we present the modifications we brought to the original SARSIFT algorithm. An exhaustive description as well as a comparison of the performances of the modified SAR-SIFT compared to SIFT and simple 2D correlation based on sliding window is given in Dubois (2015). In Sect. 3.3, we explain the chosen methodology for analysing qualitatively the possibility of combining SAR-SIFT with the stereo-SAR model for generating 3D points automatically. Tests are performed on high-resolution SpotLight TerraSAR-X images, and assessed using a LiDAR-based Digital Elevation Model (DEM). Results are then presented in Sect. 4.

\section{Methodology}

In this section, the methodology of the generation and accuracy assessment of the TPs using SAR-SIFT are explained. First, we present our overall workflow. Second, we explain the individual steps in detail. Particular attention is drawn to the accuracy assessment, which is the focus of this paper, using both single and multiple image pairs for TP generation.

\subsection{Overall Workflow}

Figure 1 shows our overall workflow. It consists of two main steps: fully automatic TP extraction and accuracy assessment. For the TP extraction, a modified SAR-SIFT is performed on each single radargrammetric image pair, to find similar points in both images. Then, using the matched points and the radargrammetric configuration defined by both images, the 3D point coordinates are retrieved. In our work, a modified version of the SAR-SIFT algorithm presented in Dellinger et al. (2015) is used (cf. Sect. 3.2). The accuracy 


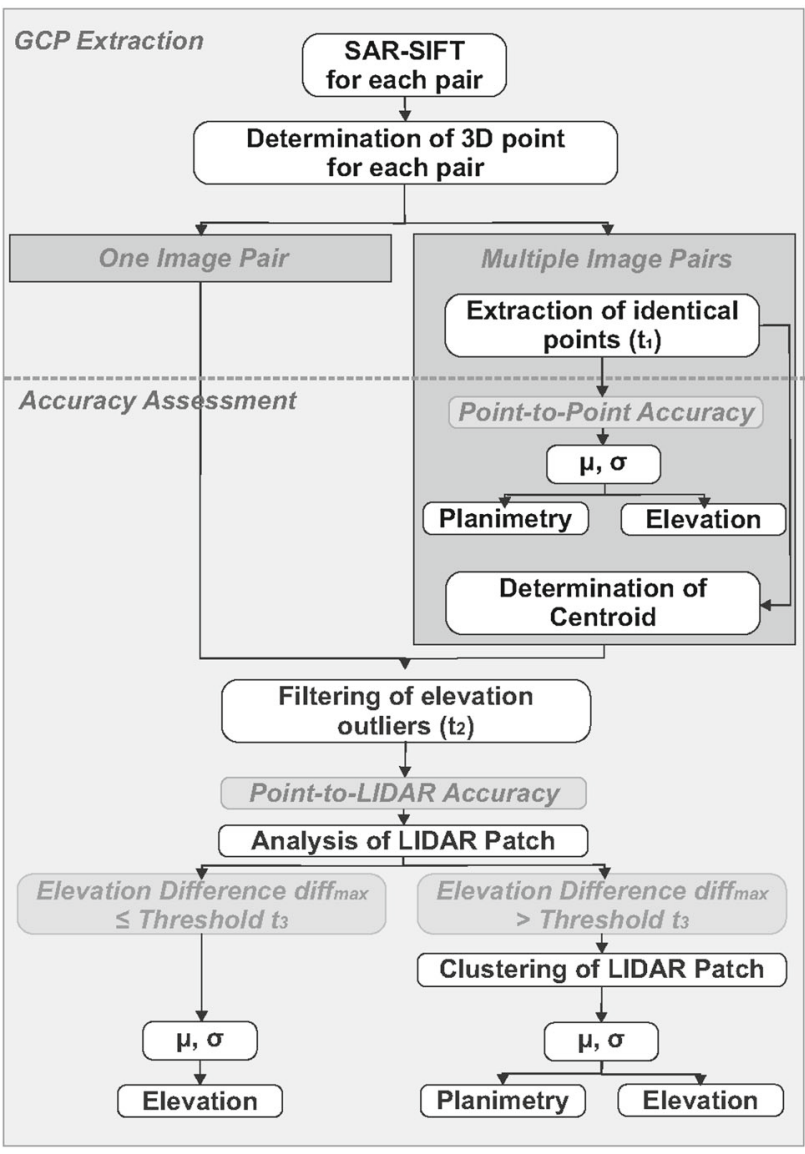

Fig. 1 Workflow of the extraction and accuracy assessment of Tie Points (TPs)

of the extracted points is then assessed to determine if they are good enough as TPs or even as GCPs. To analyse the robustness of the TP extraction and investigate the quantity of images needed for sufficient accuracy, we apply different workflows depending on whether only one or multiple radargrammetric image pairs are available. The configurations considered in our work are shown in Fig. 2. For both cases, similar intersection angle of the radargrammetric configurations is considered, and the same image serves as the master. Multiple image pairs are produced using a stack of slave images, taken from the same incidence angle, each of them being processed independently with the same master image (Fig. 2b). The use of multiple image pairs implies that one needs to find common points between the different pairs. In this context, the $3 \mathrm{D}$ points are determined three times, each time between the master and one slave image. Of course, the 3D points estimated for each image pair do not have exactly the same coordinates. Thus, only those conjugate 3D points are kept whose positions approximately coincide (based on a threshold $t_{1}$ ). To eliminate obvious outliers that correspond to wrong matches, a threshold $t_{2}$ is set on the point elevation value. This threshold is applicable also in the case of only

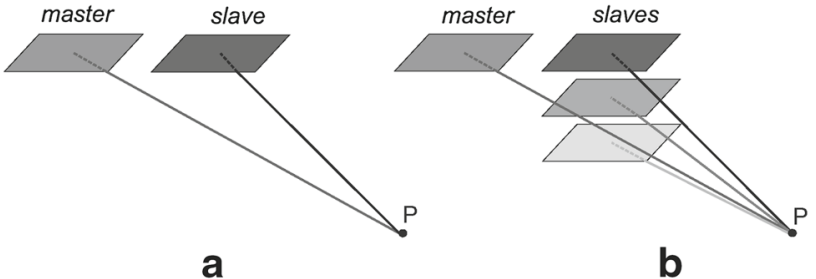

Fig. 2 Possible radargrammetric configurations for TP extraction: a one image pair and $\mathbf{b}$ multiple image pairs

using one image pair. It is set by comparing the extracted point height to a coarse surface model, which is assumed to be given, e.g., from SRTM, photogrammetry, LiDAR, or map data. Then, a point-to-point precision can be determined between corresponding points of the different pairs, and for multiple images, the 3D centroid coordinates are calculated for such conjugate 3D points, which takes into account slight 3D point displacement between the different image pairs.

An assessment using external LiDAR data can be performed for both single and multiple image pairs, i.e., a Point-to-LiDAR accuracy is determined for each extracted 3D TP. To this end, the neighbourhood of each point in the LiDAR data is analysed to define if the point is situated in a flat area or at a step edge. In this later case, the LiDAR data are clustered within a patch to exclude LiDAR points that are far beyond the height range of the determined 3D TP. For this purpose, a third threshold $t_{3}$ is introduced. Finally, a planimetric assessment is performed by determining the horizontal distance of the TP to the nearest edge (i.e., height discontinuity) of the LiDAR data. The underlying assumption is that the TPs mostly lie on double-bounce structures in the SAR image, as they often appear at height jumps of the reflecting surface. In case no particular height difference occurs within the considered point neighbourhood in the LiDAR data, only the elevation is assessed, as there is no indication about the correct planimetric point position. The step of accuracy assessment is detailed in Sect. 3.3.

\subsection{TP Extraction}

In this section, the extraction of the TPs is explained in more detail. First, modifications of the SAR-SIFT algorithm for our goal are explained. This is followed by the description of the 3D stereo model that is used for the 3D point determination.

The extraction of SAR-SIFT points is based on the approach of Dellinger et al. (2015). Besides the introduction of a new method for gradient computation, a circular neighbourhood is used for both orientation assignment and descriptor extraction. In this work, we chose to replace the latter by a square neighbourhood, as in the original SIFT (Lowe 1999). Indeed, our test area is situated in an urban environment, which presents numerous linear structures, e.g., 
buildings, fences, and other man-made objects. Keypoints are mainly situated on those linear, bright, man-made objects, or at their outline. The defined gradients follow the orientation of these objects. Therefore, changes of image intensity and gradient values around the extracted keypoints can be better assessed using a square neighbourhood that defines linear areas of equal dimension on both sides of the object. The dimension of half the square neighbourhood is defined by $8 \sigma$, whereby $\sigma$ is the corresponding image scale defined in Dellinger et al. (2015). Furthermore, the matching criterion used in this work relies on NNDR, which shows good performance despite the difference of incidence angles between the images. Besides, both images are calibrated during a preprocessing step, to reduce the radiometric influence of the difference of incidence angles. This new method is presented in more details in Dubois (2015), where it is also compared to the standard SIFT approaches. Although a direct comparison with the original SAR-SIFT presented in Dellinger et al. (2015) could not be performed, since the source code of the original SAR-SIFT algorithm is not available to the public, the results for the modified SAR-SIFT presented in Dubois (2015) fit very well. It is shown that the percentage of matched keypoints is at least as good as the one mentioned in Dellinger et al. (2015) and even better for the test site used in this paper. Furthermore, the obtained 2D accuracy after matching is also better for the test scene. This indicates that the proposed modified SAR-SIFT outperforms the original algorithm in urban areas.

The matched keypoints are transformed into 3D object space using radargrammetry. In contrast to Dellinger et al. (2015), no filtering of false matches using RANSAC is carried out, because our goal is not to robustly estimate the $2 \mathrm{D}$ transformation between the master and slave images. We aim at keeping a maximum of potential 3D TPs and filtering them afterwards based on their 3D accuracy.

The determination of the 3D point coordinates is performed using the sensor-object stereo model defined in Leberl (1990). The position of a specific point is defined in a single SAR image by its Doppler frequency and its range. The locus of equal Doppler frequency is represented as a cone and the one of equal range as a sphere. Therefore, the intersection circle between the cone and the sphere represents the potential positions of the point. This can be reduced into a set of the two fundamental SAR slant-range equations expressed by

$$
\left\{\begin{array}{l}
|P-S|=r_{p} \\
\dot{S} \cdot(P-S)=0
\end{array}\right.
$$

where $P$ and $S$ are the point and the sensor position vectors, respectively. $\dot{S}$ is the sensor velocity and $r_{p}$ is the range of point $P$. All the model parameters are provided in the product metadata and only the point position $P=\left(X_{p}, Y_{p}, Z_{p}\right)$ is unknown. Considering two acquisitions in a radargrammetric configuration, two circles arise, which intersect at two different positions, only one of which is plausible. Considering at least two images, the system is overdetermined, presenting four equations for three unknowns. In this work, a standard least square method (LSM) was used for retrieving the correct 3D point coordinates, as in Nascetti et al. (2015b).

\subsection{Accuracy Assessment}

After the 3D point coordinates have been determined, it is necessary to evaluate their accuracy to assess their capability for serving as reliable TP or - even more-as GCP in the case that 3D object coordinates are available. Here, depending on the considered configuration-one or multiple pair(s) different tests have been performed. This is presented in the following subsections.

\subsubsection{One Image Pair}

The configuration using a single image pair is represented in Fig. 2a, where only one slave image is used. All points resulting from the SAR-SIFT matching and remaining after applying $t_{2}$ (set to $1-2 \mathrm{~m}$ ) are considered as potential TPs/GCPs. Their accuracy is assessed using LiDAR data as external source for evaluation. For this purpose, a small LiDAR patch is extracted around each 3D point and the maximum LiDAR height difference diff max $_{\text {ax }}$ within the patch is calculated. If diff $_{\max }$ is greater than a certain threshold $t_{3}$, set to $3 \mathrm{~m}$ in this work, the patch is considered to include a height discontinuity, e.g., a building corner or an edge. As mentioned above, we assume that the planimetric position of the extracted 3D point corresponds to the $2 \mathrm{D}$ position of the double-bounce structure, which usually generates the corresponding image feature. Please note that these structures can appear at the bottom of facades but also at the roof line. As both features can show strong reflections, they are favoured by the SAR-SIFT keypoint detection (although the SIFT algorithm should favour corners and not edges). The speckle effect may influence this as well, but even more artifacts are given by strong backscatter fluctuations of neighbouring SAR pixels, e.g., resulting from metallic or other specific structures in the SAR image. This may lead to the situation that a point is not situated exactly at a corner or an edge, but a few pixels aside. Such a configuration is illustrated in Fig. 4b, d, e. In the case where two distinct height values are detected within the LiDAR patch, the later is separated in two different clusters $C_{\text {bot }}$ (corresponding to the ground height, in our assumption) and $C_{\text {top }}$ (corresponding to the roof height, in our assumption), using the $k$-means classification algorithm. $C_{\text {min }}$ is that particular cluster which presents the minimal height difference to the corresponding estimated 3D point 


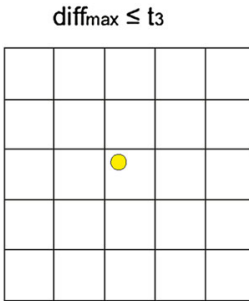

a

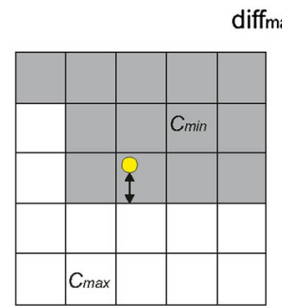

b
Fig. 3 Schematic representation of the potential position of a TP with respect to the LiDAR data: $\mathbf{a}$ on flat area and $\mathbf{b}$ near a corner or edge

$Z_{p}$. These clusters are important for the following elevation accuracy.

The planimetric accuracy of the 3D point is determined by calculating its distance to the nearest height discontinuity in the LiDAR data. This distance is determined with subpixel accuracy, considering the minimal distance between the determined 3D point and the nearest border of edge pixels in the LiDAR patch (Fig. 3b). For future work, it would be interesting to consider the LiDAR point cloud instead of the subsequently generated raster grid, as it would allow an analysis on the basis of point-to-point distances and, thus, permit a more realistic evaluation.

The elevation accuracy is calculated from the height difference between the height of the estimated 3D point and the median height of the patch, i.e., the median height of $C_{\min }$. It is important to notice that if the point is situated at an intermediate height position (for example, on a balcony along a building facade), it will induce an error that influences the estimated overall quality, as it will be either compared to the roof height or to the ground height. Without knowing the detailed structure of the scene or even the semantics of the objects, there is no possibility to compare such points correctly. However, we assume that really strong backscatters, whose selection is favoured by the algorithm, are situated either at the footprint of a building (due to double-bounce) or on the roof (due to special edge structures as, e.g., metallic rain gutter). Points situated in a LiDAR patch presenting different heights are, therefore, more reliable for evaluating the planimetric accuracy. Despite this assumption, the point quality is sufficient, as will be shown below.

In the case where diff max $_{\text {ax }}$ stays smaller than the threshold $t_{3}$, the LiDAR patch is considered to correspond to a flat area, and the potential TP is situated on flat terrain (Fig. 3a). In this case, no planimetric distance to a reference feature can be defined, as there is no distinct edge or corner. Only an estimation of the elevation accuracy is performed, measuring the difference between the point height and the median height of the LiDAR patch.

The overall accuracy is finally delineated by calculating the mean and standard deviation of the planimetric and elevation accuracy over all TPs.

\subsubsection{Multiple Image Pairs}

Considering multiple image pairs, the methodology is slightly different. This configuration is represented Fig. 2b, whereby the correspondence based on SAR-SIFT is performed between each slave image and the master image. For such configurations, multiple sets of $3 \mathrm{D}$ points are generated from the
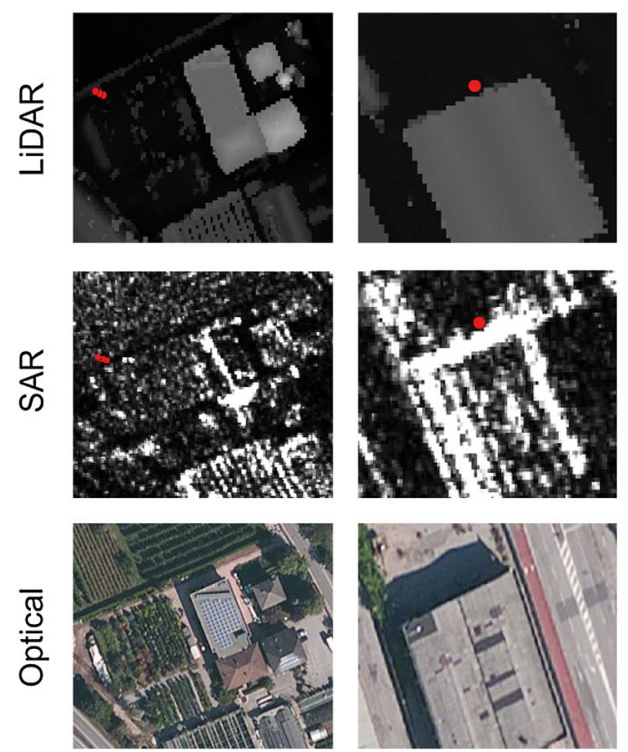

a
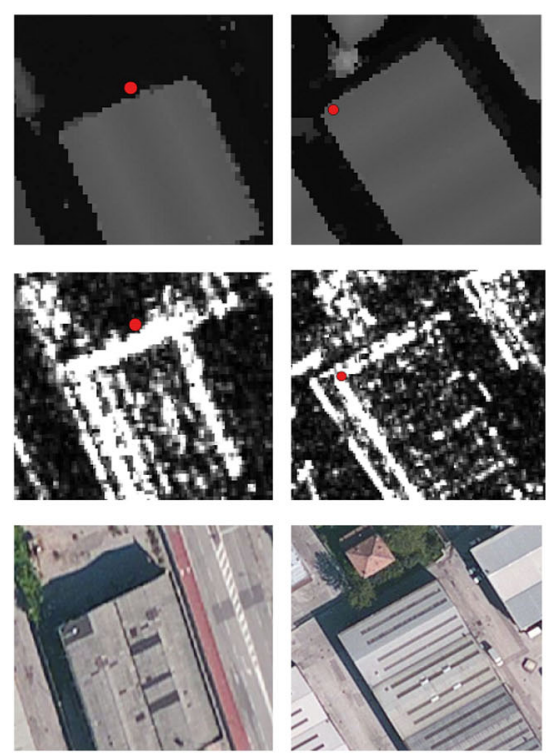

b

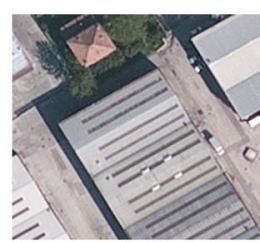

C
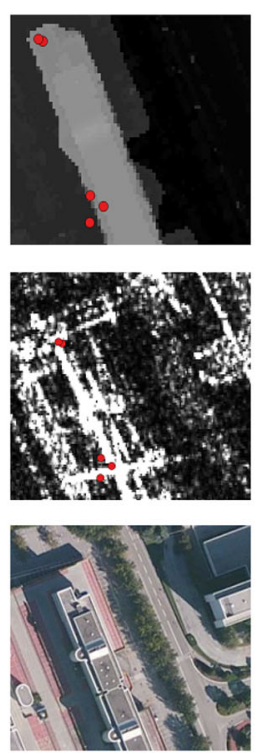

d
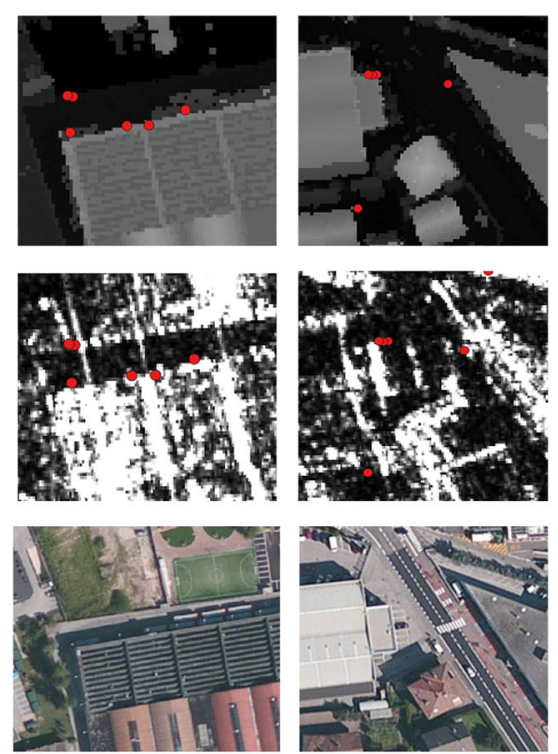

e
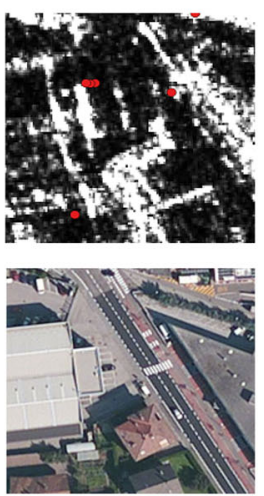

f

Fig. 4 Extracted 3D points represented in LiDAR and SAR data. Optical image (Bing Maps) is shown for better comparison: a, e, f points situated on flat area and $\mathbf{b}-\mathbf{f}$ points situated on building corners or edges 
individual 2D correspondences. Before assessing the final point accuracy, identical 3D points, i.e., those points that should be derived from truly corresponding features over all images, are selected. For this purpose, we apply a threshold $t_{1}$ (set to $2.5 \mathrm{~m}$ ) to the horizontal distance between all conjugate points. A point-to-point precision can subsequently be determined, where the mean distance and standard deviation between the identical 3D points of the multiple pairs are calculated. Results of this assessment are shown in Sect. 4. As in Sect. 3.3.1 also a comparison with an approximate surface (using $t_{2}$ ) is possible. This can be neglected if the multiple 3D points coincide. To evaluate the absolute accuracy of the extracted points using the LiDAR data by applying $t_{3}$, the centroid coordinates are determined for the conjugate identical points. To do this robustly, the respective median value of the $X, Y$, and $Z$ coordinates is used for the final centroid value. This allows to filter potential outliers that would otherwise influence the point coordinates and corrupt the final accuracy. With the centroid coordinates, the point-to-LiDAR accuracy the is assessed as in the case of using only one image pair.

Depending on the final assessment one may also choose if certain TPs may serve as GCPs.

\section{Results}

In this section, results of the accuracy assessment are shown, and the suitability of the SAR-SIFT combined to a 3D stereoSAR model for TP extraction is discussed.

\subsection{Test Area}

We considered two high-resolution SpotLight TerraSAR$\mathrm{X}$ data sets, acquired on ascending and descending orbits, respectively. LiDAR data from last-pulse response are available for these data sets, given in a $1 \mathrm{~m} \times 1 \mathrm{~m}$ raster with a vertical accuracy of $0.2 \mathrm{~m}$. Table 1 resumes the considered acquisition configurations. The acquired scene represents a partly urbanised and industrial area of Trento city centre, Italy. The considered area is of about $600 \mathrm{~m} \times 600 \mathrm{~m}$. For both orbits, the acquisition chosen to be the master image presents the smallest incidence angle. The intersection angle between master and slave(s) is of about $10^{\circ}$. For a single image pair, the first slave image in Table 1 is paired with the master image. For multiple image pairs, all slave images of the considered scene are taken into account.

\subsection{Tie Point Extraction}

In this work, we extracted TPs for the ascending and descending configurations separately. The dimensions of the LiDAR patch are set to $5 \times 5$ pix. Depending on the chosen config-
Table 1 Acquisition parameters

\begin{tabular}{lll}
\hline Name & TerraSAR-X 1 & TerraSAR-X 2 \\
\hline Orbit & Ascending & Descending \\
Master & $44^{\circ}, 16-01-2011$ & $39^{\circ}, 14-01-2011$ \\
Slaves & $53^{\circ}, 21-01-2011$ & $50^{\circ}, 20-01-2011$ \\
& $53^{\circ}, 05-04-2012$ & $50^{\circ}, 07-01-2012$ \\
& $53^{\circ}, 03-04-2013$ & $50^{\circ}, 02-04-2013$ \\
\hline
\end{tabular}

Table 2 TP accuracy for a single image pair

\begin{tabular}{lll}
\hline Name & TerraSAR-X 1 & TerraSAR-X 2 \\
\hline Number of common points & 289 & 380 \\
Point-to-LiDAR subpixel accuracy & & \\
Elevation & & \\
$\mu$ & 2.11 & 1.79 \\
$\sigma$ & 1.84 & 1.68 \\
Number of points for planimetry & 142 & 172 \\
Point-to-LiDAR subpixel accuracy & & \\
Planimetry & & \\
$\mu$ & 1.01 & 0.95 \\
$\sigma$ & 0.56 & 0.59 \\
Elevation & & \\
$\mu$ & 1.79 & 1.63 \\
$\sigma$ & 1.51 & 1.63 \\
Number of points flat area & 147 & 208 \\
Point-to-LiDAR subpixel accuracy & & \\
Elevation & & 1.93 \\
$\mu$ & 2.41 & 1.70 \\
$\sigma$ & 2.08 & \\
\hline
\end{tabular}

uration (one pair or multiple pairs), different accuracies are observed.

\subsubsection{One Image Pair}

Table 2 shows the results of the TP accuracy for a single image pair. A total number of 301 and 390 points are found by image matching for the two data sets, respectively. About half of them (142 and 172, respectively) are situated near corners or edges, allowing planimetric assessment for those points. The planimetric accuracy of the TPs reaches about $1 \mathrm{~m}$, which corresponds to the resolution of the sensor. The height accuracy is slightly worse, with a mean height difference of the extracted points to the LiDAR data of about $2 \mathrm{~m}$. This result has to be considered carefully, as the elevation accuracy differs about $0.5 \mathrm{~m}$ between both data sets, in flat areas $(2.41 \mathrm{~m}$ for TerraSAR-X 1 and $1.93 \mathrm{~m}$ for TerraSAR$\mathrm{X} 2$ ). 
The points situated near corners or edges show a slightly better elevation accuracy than those situated on flat terrain, for both TerraSAR-X data sets $(1.79$ and $1.63 \mathrm{~m}$, respectively). Besides, the standard deviation of the height difference is similar to the mean accuracy, about $2 \mathrm{~m}$. This can be explained considering that some points detected in flat areas might be situated on fences or other small objects that are not represented in the LiDAR data, inducing an offset in the height calculation. This case occurs, for example, in Fig. $4 \mathrm{a}$, where the point seems to be situated on a fence at the border of the vineyard. This changes the estimated overall elevation accuracy, but not the quality of TP itself, and not the planimetric accuracy. Despite such points, the overall accuracy of the investigated TPs remains very good.

\subsubsection{Multiple Image Pairs}

Table 3 shows the results for multiple image pairs. Here, only few common points are extracted $(23$ and 22 for ascending-TerraSAR-X 1 - and descending-TerraSAR$\mathrm{X} 2$-respectively). The point-to-point precision shows that the distance between the retained identical points of all images is of about $1 \mathrm{~m}$, with a small standard deviation. After estimation of the centroid, it is observable that half of the centroids are situated near step edges, similar to the assessment based on a single image pair. These points are used for the planimetric assessment. The point-to-LiDAR evaluation using the centroids shows a slightly better planimetric accuracy than for single image pairs: 0.70 and $0.86 \mathrm{~m}$ for the first and second data sets, respectively. The standard deviation is also very low, of about half a meter. The elevation accuracy using multiple image pairs is similar to the one obtained with a single image pair. Considering the data set TerraSAR-X 1, it seems that the elevation accuracy for points situated on flat areas improves when more image pairs are used. However, this result cannot be verified for the data set TerraSAR-X 2 . We will investigate this difference in more detail in future work.

As a conclusion, the use of multiple image pairs implies a drastic diminution of the number of TPs, yet it shows only a slight improvement in terms of point accuracy. This may be contributed to the geometric configuration, which does not change very much for multiple images, even if outliers can be removed much easier. In future work, it would be interesting to observe if this is also true when applying a more sophisticated approach for multiple images like a radargrammetric "bundle" adjustment.

In general, the determined TPs are very accurate in planimetry, showing less than $1 \mathrm{~m}$ difference to the reference. The elevation accuracy is a little bit worse, showing about $2 \mathrm{~m}$ difference to the reference for both single and multiple image pairs. This accuracy is sufficient for a large amount of TPs, e.g., for application related to georeferencing
Table 3 TP accuracy for multiple image pairs

\begin{tabular}{|c|c|c|}
\hline & TerraSAR-X 1 & TerraSAR-X 2 \\
\hline Number of common points & 23 & 22 \\
\hline \multicolumn{3}{|l|}{ Point-to-point precision } \\
\hline \multicolumn{3}{|l|}{ Planimetry } \\
\hline$\mu$ & 1.28 & 1.07 \\
\hline$\sigma$ & 0.36 & 0.33 \\
\hline \multicolumn{3}{|l|}{ Elevation } \\
\hline$\mu$ & 1.34 & 0.96 \\
\hline$\sigma$ & 0.62 & 0.53 \\
\hline \multicolumn{3}{|l|}{ Point-to-LiDAR subpixel accuracy } \\
\hline \multicolumn{3}{|l|}{ Elevation } \\
\hline$\mu$ & 1.95 & 2.09 \\
\hline$\sigma$ & 1.40 & 1.98 \\
\hline Number of points for planimetry & 14 & 12 \\
\hline \multicolumn{3}{|l|}{ Point-to-LiDAR subpixel accuracy } \\
\hline \multicolumn{3}{|l|}{ Planimetry } \\
\hline$\mu$ & 0.70 & 0.86 \\
\hline$\sigma$ & 0.51 & 0.56 \\
\hline \multicolumn{3}{|l|}{ Elevation } \\
\hline$\mu$ & 1.82 & 1.99 \\
\hline$\sigma$ & 1.22 & 1.74 \\
\hline Number of points for flat area & 9 & 10 \\
\hline \multicolumn{3}{|l|}{ Point-to-LiDAR subpixel accuracy } \\
\hline \multicolumn{3}{|l|}{ Elevation } \\
\hline$\mu$ & 2.15 & 2.21 \\
\hline$\sigma$ & 1.70 & 2.33 \\
\hline
\end{tabular}

Table 4 Influence of the acquisition configuration on the number of matches and achieved accuracy—from Dubois (2015)

\begin{tabular}{lll}
\hline $\begin{array}{l}\text { Difference of } \\
\text { incidence angles }\end{array}$ & $\begin{array}{l}\text { Number of } \\
\text { matches }\end{array}$ & $\begin{array}{l}\text { 2D accuracy after } \\
\text { coregistration } \\
(\mathrm{m})\end{array}$ \\
\hline $10^{\circ}$ descending & 601 & 0.3 \\
$11^{\circ}$ ascending & 300 & 0.2 \\
$13^{\circ}$ descending & 111 & 0.3 \\
$14^{\circ}$ ascending & 199 & 1 \\
$23^{\circ}$ descending & - & - \\
$25^{\circ}$ ascending & - & - \\
\hline
\end{tabular}

of SAR images local enhancement of DEM heights or linking radargrammetric $3 \mathrm{D}$ points to GPS measurement that are usually used as GCPs. Furthermore, compared to existing SAR-based DEMs, the absolute TP position is very accurate. As an example, the TanDEM-X mission aims at creating a DEM with a relative height accuracy of $2 \mathrm{~m}$, but with $10 \mathrm{~m}$ absolute height accuracy, in a $12 \mathrm{~m}$ grid (Gonzalez et al. 2009). Here, we obtain a sparse point distribution, with higher absolute accuracy, which could complement locally 
the global DEM estimate. The knowledge of more accurate points is especially important in urban areas, where the DEM quality decreases and could be replaced pointwise to a local DSM.

At this point, it seems interesting to investigate the influence of the difference of incidence angles between master and slave image. This question was already discussed thoroughly in Dubois (2015) for the coregistration of stereo images using the modified SAR-SIFT; therefore, we recall only the most important conclusions for this paper. Table 4 shows the number of matches found for different configurations (before coregistration) and the corresponding accuracy (2D) of the subsequent coregistration. For very different incidence angles $\left(>20^{\circ}\right)$, the proposed approach does not work anymore. Besides, the higher the difference of incidence angles, the less matches are obtained and the less robust and accurate is the result. Similar conclusions could be formulated by considering the achieved 3D accuracy using multiple image pairs (cf. Table 3). There, it seems that the achieved 3D accuracy of the final TPs is better for the configuration presenting the smallest difference of incidence angles between master and slave (TerraSAR-X 1). Using only a single image pair, contradictory conclusions would be obtained. Please notice that the difference of incidence angles between both data sets (TerraSAR-X 1: $9^{\circ}$ and TerraSAR-X 2: $11^{\circ}$ ) is very small and that these conclusions can change slightly considering the data set.

\section{Conclusion}

In this paper, we presented a new application of the SARSIFT described in Dellinger et al. (2015), focusing on 3D point determination. Considering several configurations, we analysed the 3D accuracy of the extracted points. We showed that the obtained $3 \mathrm{D}$ point accuracy is very good, slightly better in planimetry than in elevation. The use of multiple image pairs only improves the point accuracy of a minor amount-at the cost of a significant reduction of the number of determined TPs. Therefore, the use of one single image pair would be preferred, as it is also available in a shorter time span. The absolute height accuracy is higher as the one obtained by current DEM spaceborne mission as TanDEM-X, proving that this method could be used locally to enhance existing DEMs. The point density around structures is as high as in flat areas, showing the capability of the determined TPs to replace or enhance locally DEMs. Furthermore, the obtained accuracies are even better using this automatic method than using a manual approach as in Koppe et al. (2012). Considering the planimetric accuracy, it has been assumed that the point of interest was situated at structures like building edges. However, the extracted points could correspond to a strong scatterer situated farther away on the roof. Such an analysis could, therefore, benefit of a more detailed LiDAR information.

In future work, a comparison using the DEMANAL software (Jacobsen 2004) will be performed. Some preliminary tests showed some discrepancies with our analysis. The main reason for the discrepancies is related to the fact that DEMANAL uses a point-to-point 2.5D assessment between LiDAR interpolated slope and extracted point, instead of a LiDAR patch as in this work. The later provides a full 3D accuracy. However, the DEMANAL software could help to deal with strong backscatters situated on building facades.

Moreover, an optimisation for estimating the real point position would be possible. For example, one could look for the nearest edge in range direction, towards the sensor. Even more optimal for the accuracy evaluation would be to use a ray tracing simulation, where the exact point position could be retrieved.

Furthermore, we want to use the SAR-SIFT on an image stack acquired with the same incidence angle and test its ability of extracting persistent amplitude scatterers (PAS). Such PAS could be used in approaches like stereo image geodesy, presented in Capaldo et al. (2014), which aim at calculating absolute land subsidence by radargrammetry.

Finally, this approach could be extended to new sensors, as Sentinel-1, to test the capacity of the SAR-SIFT of extracting TPs at lower resolution, over wide rural areas.

Acknowledgements The authors would like to thank the Karlsruhe House of Young Scientist (KHYS) for giving C. Dubois the financial support to stay at DICEA for the development of this methodology. The authors are indebted to Prof. UWE SOERGEL and DLR for supplying the TerraSAR-X SpotLight imagery used in this work in the frame of the project Evaluation of DEM derived from TerraSAR-X data organised by the ISPRS Working Group VII/2 SAR Interferometry.

\section{References}

Capaldo P, Fratarcangeli F, Nascetti A, Mazzoni A, Porfiri M, Crespi $M$ (2014) Centimeter range measurement using amplitude data of TerraSAR-X imagery. Int Arch Photogramm Remote Sens Spatial Inf Sci XL(7): 55-61

Chureesampant K, Susaki J (2014) Automatic GCP extraction of fully polarimetric SAR images. IEEE Trans Geosci Remote Sens 52(1):137-148

Dellinger F, Delon J, Gousseau Y, Michel J, Tupin F (2015) SAR-sift: a sift-like algorithm for SAR images. IEEE Trans Geosci Remote Sens 53(1):453-466

Dubois C (2015) Interferometric synthetic aperture RADAR and radargrammetry towards the categorization of building changes. $\mathrm{PhD}$ thesis. Karlsruhe, Karlsruher Institut für Technologie (KIT), Dissertation. 2015

Fjortoft R, Lopes A, Marthon P, Cubero-Castan E (1998) An optimal multiedge detector for SAR image segmentation. IEEE Trans Geosci Remote Sens 36(3):793-802

Gonzalez JH, Bachmann M, Böer J, Fiedler H, Krieger G, Zink M (2009) TanDEM-X mission and DEM accuracy. In: Proceedings 
of wave propagation in communication, microwaves systems and navigation, WFMN 2009

Jacobsen K (2004) Analysis of digital elevation models based on space information. In: EARSEL symposium, Dubrovnik, Croatia

Koppe W, Wenzel R, Henning S, Janoth J, Hummel P, Raggam H (2012) Quality assessment of TerraSAR-X derived ground control points. In: IEEE international geoscience and remote sensing symposium, pp 3580-3583

Leberl FW (1990) Radargrammetric image processing. Artech House, Boston

Liu J-Z, Yu X-C (2008) Research on SAR image matching technology based on SIFT. Int Arch Photogramm Remote Sens Spatial Inf Sci XXXVII(B1):403-408

Lowe DG (1999) Object recognition from local scale-invariant features. In: IEEE international conference on computer vision, vol 2, pp $1150-1157$

Mittermayer J, Wollstadt S, Prats-Iraola P, Scheiber R (2014) The TerraSAR-X staring spotlight mode concept. IEEE Trans Geosci Remote Sens 52(6):3695-3706
Nascetti A, Capaldo P, Fratarcangeli F, Mazzoni A, Crespi M (2015a) Monitoring ground displacements at centimeter level exploiting TerraSAR-X range measurements. In: IEEE international geoscience and remote sensing symposium, pp 1401-1404

Nascetti A, Capaldo P, Porfiri M, Pieralice F, Fratarcangeli F, Benenati L, Crespi M (2015b) Fast terrain modelling for hydrogeological risk mapping and emergency management: the contribution of high-resolution satellite SAR imagery. Geomat Nat Hazards Risk 6(5-7):554-582

Reinartz P, Müller R, Schwind P, Suri S, Bamler R (2011) Orthorectification of VHR optical satellite data exploiting the geometric accuracy of TerraSAR-X data. ISPRS J Photogramm Remote Sens 66(1):124-132

Suri S, Schwind P, Uhl J, Reinhartz P (2010) Modifications in the SIFT operator for effective SAR image matching. Int J Image Data Fusion 1(3):243-256 